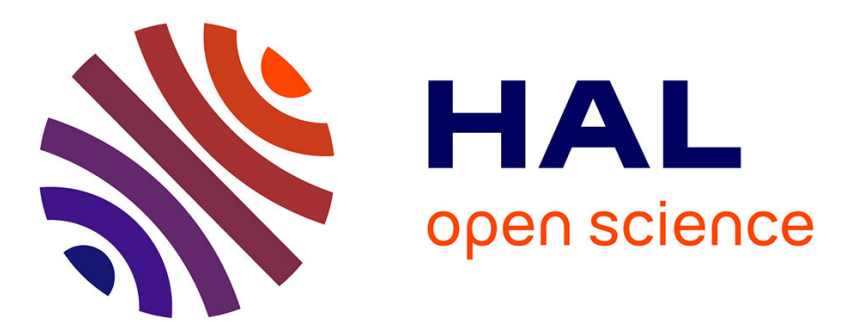

\title{
Vaccinations des patients adultes atteints d'une maladie rénale
}

\author{
Claire Trivin-Avillach, Éric Thervet
}

\section{To cite this version:}

Claire Trivin-Avillach, Éric Thervet. Vaccinations des patients adultes atteints d'une maladie rénale. Néphrologie \& Thérapeutique, 2019, 15, pp.233 - 240. 10.1016/j.nephro.2017.11.007 . hal-03486372

\section{HAL Id: hal-03486372 \\ https://hal.science/hal-03486372}

Submitted on 20 Dec 2021

HAL is a multi-disciplinary open access archive for the deposit and dissemination of scientific research documents, whether they are published or not. The documents may come from teaching and research institutions in France or abroad, or from public or private research centers.
L'archive ouverte pluridisciplinaire HAL, est destinée au dépôt et à la diffusion de documents scientifiques de niveau recherche, publiés ou non, émanant des établissements d'enseignement et de recherche français ou étrangers, des laboratoires publics ou privés.

\section{다)(1) $(5$}

Distributed under a Creative Commons Attribution - NonCommercial| 4.0 International 


\section{Vaccinations des patients adultes atteints d'une maladie rénale Immunizations for patients with kidney disease}

Claire Trivin-Avillach ${ }^{\mathrm{a}}$, Éric Thervet ${ }^{\mathrm{b},{ }^{*}}$

${ }^{a}$ Renal department, Boston University Medical Center, Boston, MA, USA

b Service de néphrologie, hôpital européen Georges Pompidou, 20 rue Leblanc, 75015 Paris, Université Paris Descartes, Paris, France

${ }^{*}$ Auteur correspondant e-mail : eric.thervet@aphp.fr

Absence de financement 


\section{Résumé}

Les patients présentant une maladie rénale chronique ont un risque infectieux accru qui peut être diminué par la vaccination. Cette susceptibilité aux infections peut être secondaire à la néphropathie comme, par exemple, dans le cadre du syndrome néphrotique, aux traitements utilisés, notamment immunosuppresseurs, ou au traitement de suppléance. De plus, l'altération de la fonction rénale entraîne une diminution de la réponse vaccinale pouvant nécessiter une adaptation des schémas vaccinaux. Les traitements immunosuppresseurs couramment employés en néphrologie contre-indiquent l'utilisation de certains vaccins et peuvent impliquer la réalisation de sérologies préalables. Le but de cette synthèse est de proposer des pistes de calendrier vaccinal selon les caractéristiques des patients rencontrés en néphrologie et de leurs pathologies.

Mots clés : glomérulopathie ; immunité ; infection ; insuffisance rénale chronique ; vaccin ; transplantation

\section{Abstract}

Chronic kidney disease (CKD) is associated with significant infectious complications leading to adverse health outcomes. This increased susceptibility to infection can be related to the nephropathy itself as observed in nephrotic syndrome, to the treatment especially in situations requiring immunosuppressive drugs or related to dialysis. Despite a less effective response to vaccination, some data emphasize similar benefits from immunization among people with CKD to the general population. However some situations encountered in nephrology require adaptation of immunization practices. The aim of this review is to provide a synthesis of the existing guidelines for immunization in the field of nephrology.

keywords: chronic kidney disease; immunity; immunization; infection; glomerulopathy; transplantion

\section{Introduction}

Les patients atteints d'une maladie rénale chronique présentent un risque infectieux accru. Ce risque peut être secondaire soit à la maladie rénale par elle-même (en cas de syndrome néphrotique, par exemple), soit aux traitements immunosuppresseurs, ou à une exposition particulière comme pour le virus de l'hépatite $B$ lors de la dialyse. De plus, la réduction chronique du débit de filtration glomérulaire (DFG) entraîne une altération de la réponse immunitaire qui est d'autant plus importante avec la sévérité de l'insuffisance rénale. Ces différentes raisons font de la vaccination un enjeu capital dans la prise en charge des patients présentant une pathologie rénale.

\section{Immunité vaccinale et maladie rénale chronique}

La détérioration de la fonction rénale est associée à un milieu pro-inflammatoire marqué et un vieillissement prématuré des cellules immunitaires entraînant une altération du système immunitaire. Les conséquences cliniques sont importantes, aussi bien en termes de morbidité que de mortalité. Ces patients sont susceptibles aux infections, présentent une augmentation du risque des cancers associés aux virus et ont une réponse vaccinale altérée [1].

L'état pro-inflammatoire est probablement multifactoriel, avec une activation des cellules immunitaires, une augmentation du stress oxydatif et des cytokines pro-inflammatoires. Cet état pro-inflammatoire est en étroite relation avec le risque infectieux et le risque cardiovasculaire [2], les deux premières causes de décès du patient présentant une insuffisance rénale chronique (IRC). II est également associé à une diminution de 
l'immunité cellulaire. Par exemple, l'activation des cellules T induite par l'urémie entraîne certes une régulation positive des récepteurs des cytokines (en particulier l'interleukine [IL]-2 et le Tumor Necrosis Factor [TNF]a), mais aussi une diminution de la réponse lymphocytaire à l'IL-2 ou au TNFa (tachyphylaxie). Un milieu pro-inflammatoire avec une diminution de l'immunité cellulaire peut être responsable d'une augmentation de la prévalence des infections. Les effets sur les différents effecteurs du système immunitaire sont revus dans l'article de Betjes [1] et résumés dans le tableau 1.

Les implications cliniques sont nombreuses. Les modifications du système immun inné et adaptatif en relation avec l'urémie sont nombreuses et montrent bien les interactions entre les deux systèmes.

En ce qui concerne la vaccination, il est clair que la diminution importante des capacités de génération de cellules $T$ Helper complètement différenciées et spécifiques d'antigène en relation avec l'urémie [3] implique une diminution de la réponse vaccinale dépendante des lymphocytes T. L'absence de maintien de la réponse sérologique dans cette population pourrait être en relation avec une augmentation de l'apoptose induite par l'activation à la fois des cellules $T$ mémoires et des cellules $B$ mémoires. Les essais d'intervention thérapeutique comme l'utilisation de GM-CSF pour améliorer la réponse vaccinale n'ont pas été couronnés de succès [4].

\section{Immunité vaccinale et syndrome néphrotique}

\section{Une immunité altérée}

Les infections sont parmi les complications les plus fréquentes chez les patients présentant un syndrome néphrotique, en particulier chez l'enfant. Dans ce cas, elles sont corrélées avec des rechutes fréquentes, une cortico-dépendance et s'accompagnent d'une augmentation de la morbi-mortalité. À l'origine de ces infections, les bactéries encapsulées et entérobactéries, au premier rang desquelles Escherichia coli, sont souvent mises en cause. Plusieurs hypothèses physiopathologiques sont avancées, telles le syndrome œdémateux, les pertes urinaires des facteurs $B$ et $D$ de la voie alterne du complément, des anomalies des fonctions phagocytaires et l'effet des traitements immunosuppresseurs.

Plusieurs études ont évalué la réponse vaccinale dans le cadre du syndrome néphrotique. Une étude française a montré qu'il existe une bonne réponse sérologique antipneumococcique chez des enfants présentant un syndrome néphrotique sous fortes doses initiales de stéroïdes [5].

La vaccination contre la varicelle est bien tolérée et conserve son aspect immunogène chez les enfants avec un syndrome néphrotique, y compris s'ils sont traités par des stéroïdes un jour sur deux [6]. Compte-tenu du risque en l'absence d'antériorité de varicelle documentée, une vaccination contre la varicelle peut être proposée une fois la rémission du syndrome néphrotique obtenue, lorsque le risque est à la récidive. En cas de doute sur l'existence d'un antécédent de varicelle, une sérologie est réalisée.

En ce qui concerne la vaccination antituberculeuse, l'efficacité du BCG ne semble pas altérée en cas de syndrome néphrotique. Une étude a montré que la vaccination antituberculeuse permettait, chez les enfants, une diminution de la survenue d'infections [7]. Le syndrome néphrotique en l'absence de traitement immunosupresseur n'est donc pas une contre-indication au BCG et l'indication reste identique à celle de la population générale, à savoir les enfants âgés de moins de 15 ans à risque élevé de tuberculose [8].

\section{En pratique, les vaccins recommandés (tableau 2)}

En cas de syndrome néphrotique, les recommandations actuelles proposent [8] :

- une double vaccination pneumococcique par une première dose de vaccin conjugué 13valent (VCP13), suivie d'une seconde injection à 8 semaines de vaccin non conjugué 23- 
valent (VPP23). Un rappel dans un délai d'au moins 5 ans par le vaccin non conjugué peut être proposé ;

- la vaccination annuelle contre la grippe saisonnière ;

- l'application du calendrier vaccinal pour la population générale.

L'existence d'un syndrome néphrotique ne s'oppose pas à l'administration des vaccins vivants atténués. Toutefois, ces patients sont en général candidats à un traitement immunosuppresseur qui contre-indique ces vaccins, mais peut nécessiter certaines vaccinations de leur entourage (voir infra). Si possible, l'administration de vaccins vivants atténués doit être réalisée après rémission du syndrome néphrotique et l'arrêt des éventuels traitements immunosuppresseurs.

\section{Traitement immunosuppresseur et vaccination}

Le champ des traitements immunosuppresseurs et biothérapies utilisés en néphrologie s'est considérablement élargi au cours de ces dernières années. Selon le traitement immunosuppresseur employé et la voie immunologique ciblée, la réponse vaccinale est plus ou moins altérée [9]. Dans le lupus, par exemple, l'utilisation de stéroïdes associée au cyclophosphamide et/ou à l'azathioprine altère peu la réponse antipneumococcique [10].

De façon systématique, il est recommandé de proposer à tout patient ayant un traitement immunomodulateur la vaccination grippale saisonnière (vaccin inactivé) et la vaccination pneumococcique (schéma vaccinal en 2 doses de 13 puis 23-valent à 8 semaines) [11]. Un rappel à 5 ans du vaccin pneumococcique non conjugué peut être proposé. II convient également, dans la mesure du possible, de proposer la vaccination de l'entourage en particulier contre la varicelle en l'absence d'immunité ancienne et la grippe.

D'autres vaccins en revanche sont contre-indiqués. En cas de biothérapie, traitement immunosuppresseur ou corticothérapie (dose $>10 \mathrm{mg}$ ) jour depuis plus de 2 semaines, ou dans les 3 mois suivant une dose de charge bolus), les vaccins suivants sont contreindiqués:

- BCG ;

- fièvre jaune ;

- rougeole-oreillons-rubéole $(R O R)$;

- grippe saisonnière par voie nasale ;

- rotavirus par voie orale ;

- varicelle ;

- zona.

En cas de nécessité, notamment pour le vaccin antiamaril en zone d'endémie, un délai de 3 mois après l'arrêt de l'agent immunosuppresseur est préconisé $(6$ mois en cas de rituximab). Concernant la vaccination contre le zona, celle-ci est recommandée dans la population générale chez les sujets âgés de 65 à 74 ans, mais pas en cas d'immunodépression. II s'agit d'un vaccin vivant atténué qui contient une préparation lyophilisée d'une souche de virus varicelle-zoster. Des données sont cependant encourageantes en cas d'immunodépression. En effet, dans une série de 110 patients traités par biothérapies (y compris par rituximab), aucun patient n'a développé d'infection zostérienne dans les 6 semaines suivant la vaccination [12]. Une autre étude rétrospective portant sur une cohorte de 62 patients atteints d'hémopathie maligne suggère une bonne tolérance du vaccin contre le zona [13]. Idéalement, le vaccin est proposé avant l'initiation du traitement immunosuppresseur chez les sujets de plus de 65 ans, voire âgés de plus de 50 ans (voir le paragraphe concernant la transplantation) [14].

Certains traitements imposent des recommandations supplémentaires :

- l'éculizumab, inhibiteur de la voie terminale du complément, est utilisé entre autres dans les syndromes hémolytiques et urémiques atypiques (SHUa). De ce fait, il semble logique de proposer que tous les patients avec un SHUa soient vaccinés contre Neisseria meningitides. La vaccination devrait être réalisée aussi tôt que possible durant la maladie 
afin d'optimiser son efficacité et doit être obligatoire avant l'administration d'éculizumab. Le vaccin tétravalent (A, C, Y, W135) conjugué et le vaccin contre le sérotype $B$ doivent être utilisés. Les recommandations actuelles précisent que les patients mis sous éculizumab moins de deux semaines après vaccination doivent être traités par une antiobioprophylaxie (pénicilline orale ou macrolides). Un doute subsistant quant à l'efficacité de la vaccination chez des patients immunodéficients (transplantés, insuffisants rénaux chroniques), les recommandations françaises préconisent de continuer le traitement prophylactique antibiotique pendant toute la durée du traitement. II est également proposé de vacciner contre le pneumocoque et la grippe saisonnière les patients ayant présenté un SHU atypique pour diminuer le risque de récidive ;

- le rituximab est un anticorps dirigé contre le récepteur CD20 des lymphocytes B. Son utilisation entraîne une déplétion en lymphocytes $B$ et donc un déficit de l'immunité humorale. Son utilisation en néphrologie est large : vascularite à anticorps anti-cytoplasme des polynucléaires neutrophiles (ANCA), glomérulonéphrite extra-membraneuse, syndrome néphrotique cortico-résistant... Des études observationnelles et une étude randomisée ont montré que les réponses humorales aux vaccinations dirigées contre la grippe et au vaccin polysaccharidique contre le pneumocoque (antigènes cellules-T indépendants) sont diminuées après traitement par rituximab [15-19]. En ce qui concerne la vaccination antitétanique, cette dernière semble moins affectée par l'effet du rituximab. Les lymphocytes $B$ sont bien entendu responsables de la réponse fonctionnelle, mais le rôle des lymphocytes $T$ nécessaires à la différenciation des lymphocytes $B$ en plasmocytes semble être prépondérant dans la réponse au vaccin antitétanique [20]. Après la repopulation $\mathrm{B}$, la réponse vaccinale contre la grippe et le pneumocoque semble restaurée [21]. En complément des vaccinations ci-dessus, il est recommandé de vérifier le statut sérologique contre l'hépatite $B$ ( $\mathrm{Ac}$ anti-HBs, Ag HBs, Ac anti-HBc) avant administration de rituximab. En cas de positivité de l'anticorps anti-HBc et/ou de l'antigène HBs, une quantification de l'ADN viral doit être demandée. En cas d'infection chronique, un traitement doit être initié par un hépatologue et le traitement par rituximab est si possible reporté. En cas d'infection ancienne guérie (Ac anti-HBc+, Ac anti-HBs+, ADN VHB-), un traitement préemptif (entécavir, lamivudine) est prescrit en raison du risque de réactivation. En l'absence d'immunisation, la vaccination peut être proposée selon le schéma habituel. Pour l'administration d'un vaccin vivant atténué (voir infra), il est conseillé d'attendre un délai de 6 mois après la dernière administration de rituximab. Si un vaccin vivant atténué a déjà été administré, il faut attendre au minimum 3 semaines avant d'initier le rituximab ;

- le bortézomib est de plus en plus utilisé en néphrologie dans le cadre de la prise en charge des maladies rénales en relation avec une gammapathie monoclonale (Monoclonal Gammapathy of Renal Significance ou MGRS). La vaccination saisonnière contre la grippe est recommandée pour tous les patients atteints d'une histoire carcinologique solide ou hématologique quel que soit le type de traitement antinéoplasique, même si l'efficacité de la réponse est extrêmement variable. II existe en effet la démonstration que $70-80 \%$ des patients avec une tumeur ont une séroconversion après vaccination [22]. Compte tenu du fait que les patients avec un lymphome malin ou myélome multiple ont une réponse diminuée, un rappel de vaccination après au moins 1 mois peut être raisonnable dans cette population [11]. Quelques résultats suggèrent une meilleure efficacité lorsque la vaccination est réalisée directement après une chimiothérapie ;

- en cas de traitement immunomodulateur par immunoglobulines (ou autre produit sanguin labile, plasmaphérèse...), ce dernier peut affecter l'efficacité de la vaccination. Ceci est particulièrement problématique pour les vaccinations contre la varicelle, le zona, la rougeole, les oreillons et la rubéole. II est conseillé de respecter un délai pour la réalisation de la vaccination contre la varicelle et le ROR. II est proposé un délai de deux semaines si le vaccin vivant atténué précède l'administration d'immunoglobulines et un 
minimum de 3 mois en cas d'administration initiale des produits sanguins labiles (jusqu'à 10 mois en cas d'administration d'immunoglobulines) [11].

\section{Vaccination et maladie rénale chronique}

En cas de maladie rénale chronique, l'altération de la réponse immunitaire majore le risque infectieux (voir supra). II est donc recommandé, en complément du schéma vaccinal habituel, de proposer les vaccinations suivantes à tout patient présentant une diminution du DFG inférieure à $60 \mathrm{~mL} / \mathrm{mn} / 1,73 \mathrm{~m}^{2}$ :

- vaccination pneumococcique par le vaccin conjugué 13-valent (VCP13), suivi du vaccin non conjugué 23-valent (VPP23) à 8 semaines, puis d'un rappel par le VPP23 à 5 ans [8] ;

- vaccination annuelle contre la grippe saisonnière ;

- vaccination contre l'hépatite B (figure 1).

La vaccination contre le virus de l'hépatite B doit être anticipée en vue d'un traitement de suppléance par dialyse ou de transplantation. Le moment idéal de la vaccination ainsi que le schéma d'administration restent débattus avec le risque de diminution de la réponse en cas de vaccination tardive.

Les recommandations varient selon les pays et sociétés savantes. En France, les recommandations de la Haute Autorité de santé (HAS) de 2012 préconisent de vacciner dès le stade 3B (DFG $<45 \mathrm{~mL} / \mathrm{mn} / 1,73 \mathrm{~m}^{2}$ ). En revanche, les KDIGO 2012 proposent une vaccination à partir du stade 4 de la maladie rénale chronique (DFG $\left.<30 \mathrm{~mL} / \mathrm{mn} / 1,73 \mathrm{~m}^{2}\right)$. Il est logique de prendre en compte la progression de la maladie rénale chronique pour le "timing" de la vaccination.

En ce qui concerne le schéma vaccinal recommandé chez l'adulte insuffisant rénal, celuici comporte 4 injections intramusculaires de $40 \mu \mathrm{g}$ réalisées à $0,1,2$ et 6 mois. Les différents vaccins monovalents disponibles sont:

- Engerix ${ }^{\circledR} 20 \mu \mathrm{g}: 2$ injections à M0, M1, M2 et M6*;

- Hbvax pro ${ }^{\circledR} 40 \mu \mathrm{g}: 1$ injection à M0, M1, M2 et M6* ;

- Genhevac $B$ Pasteur ${ }^{\circledR} 20 \mu \mathrm{g}: 2$ injections à M0, M1, M2 et $M 6^{*}$;

- Fendrix $B^{\circledR} 20 \mu \mathrm{g}: 1$ injection à $0,1,2$ et 6 mois*.

Notons que les dispensations de $\mathrm{Hbvax}$ pro ${ }^{\circledR} 40 \mu \mathrm{g}$ et de Fendrix $B^{\circledR}$ sont réservés aux patients insuffisants rénaux. Fendrix $B^{\circledR}$ contient un adjuvant, AS04, agoniste du TLR4, inducteur des lymphocytes B mémoire [23].

\section{Note de bas de page}

* Dans le cadre des tensions d'approvisionnement rencontrées depuis janvier 2017 pour le vaccin Engerix B $20 \mu \mathrm{g}$, le Haut Conseil de la santé publique (HCSP) a été amené à recommander, pour les patients insuffisants rénaux, un schéma vaccinal par Hbvax pro ${ }^{\circledR} 40 \mu \mathrm{g}$ ou Fendrix $B^{\circledR} 20 \mu \mathrm{g}$ [24], en conservant les schémas posologiques indiqués cidessus. Ces derniers sont distribués uniquement dans les pharmacies à usage intérieur (PUI) autorisées à la rétrocession des établissements de santé disposant d'un centre de dialyse et sont rétrocédables aux patients ambulatoires sur présentation d'une prescription médicale.

La vaccination doit être suivie d'un contrôle sérologique 4 à 8 semaines après la dernière injection du schéma vaccinal complet. En cas de titre d'anticorps (Ac) anti-HBs < $10 \mathrm{UI} / \mathrm{L}$, les patients sont dits non répondeurs. Toutefois, si le contrôle sérologique est effectué à distance de la vaccination, ce titre peut être $<10 \mathrm{UI} / \mathrm{L}$ alors que le patient possède une immunité effective. Dans ce cas, un rappel unique suivi d'un contrôle du titre d'anticorps permet de vérifier la réponse vaccinale.

En cas de non-réponse vaccinale (titre $A c$ anti-HBs $<10 \mathrm{UI} / \mathrm{L}$ dans un délai de 4 à 8 semaines après la dernière injection du schéma vaccinal complet), il est recommandé de répéter 1 injection de $40 \mu \mathrm{g}$, suivie d'une deuxième injection en cas de persistance du 
titre inférieure à $10 \mathrm{UI} / \mathrm{L}$ [25]. La réponse vaccinale à une injection supplémentaire chez des patients non répondeurs pourrait être améliorée par l'utilisation de membrane en polyméthylmétacrylate (PMMA) chez les patients dialysés. L'hypothèse d'action est une clairance du facteur soluble CD40 par adsorption à la membrane en PMMA [26]. Le facteur soluble CD40 est un antagoniste de l'interaction CD40-CD154 essentielle à la stimulation lymphocytaire $B$.

Chez les patients dialysés non immunisés contre le virus de l'hépatite $B$, l'administration intramusculaire d'immunoglobuline anti-VHB (lg anti-VHB) est recommandée, concomitante à un schéma vaccinal complet. Les immunoglobulines spécifiques sont efficaces à $75 \%$ dans la prévention de l'hépatite $\mathrm{B}$. Lorsqu'une vaccination simultanée est réalisée, immunoglobulines et vaccin doivent être administrés en 2 points d'injection différents. L'administration concomitante d'immunoglobulines spécifiques n'altère pas l'efficacité du vaccin [27] et a été approuvée dans les situations de prévention postexposition au virus de l'hépatite $B$ [28]. En effet, le vaccin est réalisé à partir d'un antigène recombinant. En revanche, l'efficacité de vaccins constitués de virus vivants atténués tels que le ROR ou le vaccin contre la varicelle peut être diminuée. Après administration d'lg anti-VHB, il est conseillé d'attendre un intervalle d'au moins 3 mois avant de réaliser ce type de vaccins. Si le patient a reçu des vaccins constitués de virus vivants atténués (ROR, varicelle), le laboratoire qui commercialise les Ig anti-VHB dans cette indication en France recommande un intervalle de 3 à 4 semaines avant administration d'lg anti-VHB. Si l'administration d'immunoglobulines spécifiques est nécessaire avant, une nouvelle vaccination doit être effectuée 3 mois après cette administration d'lg anti-VHB. En cas de non-réponse à une vaccination bien conduite, les injections d'lg anti-VHB doivent être répétées tous les 2 mois à la posologie de 8 à $12 \mathrm{Ul} / \mathrm{kg}$, sans dépasser $500 \mathrm{Ul}$.

Une surveillance annuelle du titre des anticorps anti-HBs est recommandée avec une injection de rappel lorsque celui-ci est inférieur à $10 \mathrm{UI} / \mathrm{L}$ [8].

\section{Vaccination et transplantation rénale}

La vaccination chez le patient transplanté est extrêmement importante pour prévenir des infections graves, des pertes de greffon et des décès. L'optimisation de la vaccination doit passer par une stratégie aussi précoce que possible au cours de la maladie rénale chronique afin de permettre une maximisation de son efficacité, d'autant que les réponses vaccinales secondaires sont moins altérées que les réponses primaires chez les patients immunodéprimés.

Les recommandations actuelles (tableau 3) préconisent, pour le patient transplanté, une vaccination pneumococcique (schéma vaccinal en 2 doses de 13 puis 23-valent à 8 semaines), une vaccination grippale annuelle et des rappels diphtérie-tétanos-polio comme dans la population générale, auxquels il peut être associé un rappel contre la coqueluche en cas d'exposition à risque [11]. D'autres peuvent être proposées dans des cadres spécifiques (vaccination méningococcique en cas d'utilisation d'éculizumab, par exemple).

De plus, il convient de prendre en compte le type de vaccin. Les vaccins non vivants peuvent être utilisés sans risque. En revanche, les vaccins vivants (varicelle, zona, rougeole, rubéole, oreillons, rotavirus, fièvre jaune, polio oral et BCG ou fièvre typhoïde oral) sont contre-indiqués durant la période de transplantation. II est donc logique de proposer ces vaccins avant la transplantation, en respectant un délai d'au moins de 4 semaines. La question des adjuvants est au-delà de la teneur de cette mise au point. Enfin, la majorité des études a montré l'absence d'effet de la vaccination sur la production d'anticorps anti-HLA de novo. II faut noter que de nombreuses équipes préfèrent éviter une vaccination dans les 6 mois à 1 an suivant la greffe, mais surtout en raison de la diminution importante de la réponse protectrice. 


\section{Hépatite B}

Tous les patients en attente de transplantation doivent être vaccinés contre l'hépatite B. La surveillance après transplantation doit être effectuée tous les ans et un rappel effectué si le titre d'anticorps est inférieur à $<10 \mathrm{U} / \mathrm{mL}$. Plusieurs études ont montré une augmentation de la probabilité de séroconversion avec une plus forte dose de vaccin (20 $\mu \mathrm{g}$ ou $40 \mu \mathrm{g}$ contre $10 \mu \mathrm{g})$.

\section{Grippe}

Tous les transplantés rénaux doivent bénéficier, hors contre-indication, d'une vaccination annuelle contre la grippe, dont l'efficacité et l'innocuité ont été démontrées [29]. Plusieurs études ont mis en évidence une amélioration de la protection par injection intradermique comparée à une injection sous-cutanée ou intramusculaire [30,31].

\section{Pneumocoque}

La vaccination pneumococcique est recommandée pour tous les transplantés rénaux. En pratique, les patients n'ayant pas été vaccinés antérieurement devraient recevoir une simple dose de vaccin conjugué VPC13 suivi, deux mois après, d'une dose de vaccin polysaccharidique 23-valent (VPP23). Après 5 ans, un rappel par le vaccin non conjugué peut être administré. Les patients ayant déjà reçu une ou plusieurs doses de VPP23 devraient recevoir une dose de VPC13 plus d'un an après la dernière dose de VPP23. L'injection ultérieure du VPP23 doit être pratiquée avec un délai minimal de 5 ans par rapport à la date de l'injection du VPP23 [8].

\section{Varicelle et zona}

Ces vaccins vivants doivent être administrés avant la transplantation, bien que son administration semble être sans risque chez les patients avec une faible immunosuppression (stéroïde ou azathioprine seul) [32-35].

II est recommandé de vacciner contre la varicelle tous les patients sans antécédent clinique de varicelle et sans immunisation documentée par une sérologie (HCSP 2017) [8]. La vaccination comporte 2 injections séparées de 4 à 8 semaines et doit respecter un délai d'au moins 4 semaines avant la transplantation. II convient de vacciner les personnes non immunisées partageant la vie des patients transplantés. Concernant le zona, le vaccin est recommandé en France chez les sujets âgés de 65 à 74 ans en l'absence d'immunodépression, tandis que les recommandations américaines proposent une vaccination pour les patients âgés de plus de 60 ans dans la population générale et de plus de 50 ans en pré-transplantation [14]. II faut noter qu'un vaccin recombinant (Shingrix ${ }^{\circledR}$ ) a été approuvé en octobre 2017 aux États-Unis en prévention du zona chez l'adulte âgé de plus de 50 ans. Ce vaccin non vivant n'est donc pas contre-indiqué en cas d'immunodépression et pourrait être administré après la transplantation.

\section{Diphtérie-tétanos-poliomyélite et coqueluche}

Les recommandations actuelles pour la population générale comprennent un schéma vaccinal complet suivi d'un rappel diphtérie-tétanos-poliomyélite (DTP) et coqueluche à l'âge de 25 ans, puis de rappels par la triple association DTP à l'âge de 45 et 65 ans, et enfin tous les 10 ans. Toutefois, chez les patients transplantés, un rappel DTP reste recommandé tous les 10 ans [11]. En cas de voyage en zone à risque de diphtérie, des rappels plus rapprochés peuvent être proposés après dosage des titres résiduels d'anticorps.

\section{Méningocoque}

Cette vaccination est surtout recommandée en cas de situation laissant penser qu'un traitement par éculizumab sera nécessaire ou en cas de splénectomie (voir ci-dessus). 


\section{Rougeole, oreillons, rubéole}

L'utilisation de ce vaccin vivant est contre-indiquée après la greffe. L'interrogatoire doit tenter de retrouver une vaccination antérieure. II est également possible de vérifier les titres d'anticorps avant la greffe et alors de vacciner avec une ou deux doses, en particulier en cas de voyage dans des régions à risque.

\section{Human papilloma virus}

Pour la population générale, le vaccin Human papilloma virus (HPV) est recommandé chez les jeunes filles âgées de 11 à 14 ans et en rattrapage jusqu'à 19 ans. Chez les patients en attente de transplantation, le vaccin HPV est proposé dès 9 ans et jusqu'à 19 ans pour les filles et les garçons [11]. La vaccination est aussi conseillée pour les hommes ayant des relations sexuelles avec des hommes jusqu'à l'âge de 26 ans [8]. Après transplantation, l'efficacité clinique n'est pas démontrée, mais une étude a mis en évidence une réponse vaccinale de $63,2 \%, 68,4 \%, 63,2 \%$ et $52,6 \%$ pour les HPV de types $6,11,16$ et 18 , respectivement [36].

\section{Hépatite A}

La vaccination contre l'hépatite $A(\mathrm{VHA})$ est recommandée pour les patients voyageant dans des régions d'endémie, chez les hommes ayant des relations sexuelles avec des hommes, en cas d'antécédents de maladie hépatique chronique ou d'utilisation de drogue intraveineuse. L'efficacité vaccinale semble réduite chez les patients transplantés rénaux. En effet, une étude a montré un taux de séroconversion réduit à $72 \%$ versus $100 \%$ dans le groupe contrôle après un schéma vaccinal complet comprenant 2 injections à 6 mois d'intervalle. De plus, le suivi sérologique à 2 ans montrait une atténuation de la réponse vaccinale avec une persistance d'anticorps anti-VHA chez seulement $26 \%$ des patients ayant au préalable une sérologie positive [37]. II semble donc préférable de proposer une vaccination avant la transplantation.

\section{Bacille de Calmette et Guérin (BCG)}

Le BCG est contre-indiqué chez le patient transplanté ou en attente de transplantation.

\section{En cas de voyage}

La question la plus importante dans ce cadre est le vaccin de la fièvre jaune qui est un vaccin vivant et donc contre-indiqué après la greffe. Il est fortement conseillé de réaliser la vaccination avant la greffe (en particulier chez des patients qui auront probablement l'envie ou la nécessité de voyager en zone endémique). II a été montré que la vaccination avant la greffe permet de maintenir un niveau protecteur de façon prolongée après la transplantation [38]. Chez les personnes transplantées antérieurement vaccinées, il est possible de doser les anticorps neutralisants antiamarils et de considérer comme protecteur un titre d'anticorps neutralisants supérieur à $10 \mathrm{U} / \mathrm{L}$. II est alors possible d'établir un certificat de contre-indication à la vaccination autorisant le voyage en zone d'endémie. L'entourage peut être vacciné sans risque.

\section{Vaccination des sujets contacts}

Les contacts proches doivent être vaccinés de façon correcte pour permettre une protection supplémentaire des patients transplantés. II s'agit en particulier des vaccins contre la varicelle (en l'absence d'antécédent clinique de varicelle chez les sujets contacts et notamment les enfants des patients transplantés ou en attente de transplantation), la fièvre jaune en cas de situations à risque, les oreillons, la rougeole et la rubéole, et plus généralement de tous les vaccins vivants injectables en l'absence d'immunisation préalable. En revanche, des vaccins non injectables doivent être évités, ou accompagnés 
de mesures d'hygiène strictes pendant au moins deux semaines (lavage des mains, etc.). La vaccination de l'entourage contre la grippe saisonnière est également conseillée. Concernant le vaccin contre la coqueluche, il n'y a pas à l'heure actuelle de recommandation spécifique pour l'entourage du patient immunodéprimé. Le calendrier vaccinal pour la population générale prévoit un rappel à l'âge de 25 ans ou un rattrapage jusqu'à l'âge de 39 ans en l'absence de vaccination à 25 ans, et à tout âge en cas de contact étroit avec un nouveau-né [8].

\section{Conclusion}

En pratique, l'immunité vaccinale doit avoir une place entière dans la prise en charge des patients rencontrés en néphrologie, quelle que soit leur pathologie rénale. La vaccination doit être anticipée en raison de l'altération de la réponse vaccinale et des potentielles contre-indications que le patient peut présenter au cours de son parcours de soin.

\section{Conflit d'intérêts : aucun.}

\section{Références}

[1] Betjes MGH. Immune cell dysfunction and inflammation in end-stage renal disease. Nat Rev Nephrol. 2013;9:255]65.

[2] Kim JK, Hong CW, Park MJ, Song YR, Kim HJ, Kim SG. Increased neutrophil extracellular trap formation in uremia is associated with chronic inflammation and prevalent coronary artery disease. J Immunol Res. 2017;2017:8415179-10.

[3] Yoon JW, Gollapudi S, Pahl MV, Vaziri ND. Naïve and central memory T-cell lymphopenia in end-stage renal disease. Kidney Int. 2006;70:37106.

[4] Verkade MA, van de Wetering J, Klepper M, Vaessen LMB, Weimar W, Betjes MGH. Peripheral blood dendritic cells and GM-CSF as an adjuvant for hepatitis B vaccination in hemodialysis patients. Kidney Int. 2004;66:614021.

[5] Ulinski T, Leroy S, Dubrel M, Danon S, Bensman A. High serological response to pneumococcal vaccine in nephrotic children at disease onset on high-dose prednisone. Pediatr Nephrol. 2008;23:1107013.

[6] Furth SL, Arbus GS, Hogg R, Tarver J, Chan C, Fivush BA, et al. Varicella vaccination in children with nephrotic syndrome: a report of the Southwest Pediatric Nephrology Study Group. J Pediatr. 2003;142:14508.

[7] Wu HM, Tang JL, Cao L, Sha ZH, Li Y. Interventions for preventing infection in nephrotic syndrome. Cochrane Database Syst Rev. 2012;(4):CD003964.

[8] Ministère des Affaires sociales et de la Santé. Calendrier des vaccinations et recommandations vaccinales 2017. Avril 2017. http://solidaritessante.gouv.fr/IMG/pdf/calendrier_vaccinations_2017.pdf

[9] Agarwal N, Ollington K, Kaneshiro M, Frenck R, Melmed GY. Are immunosuppressive medications associated with decreased responses to routine immunizations? A systematic review. Vaccine. 2012;30:1413]24.

[10] Lipnick RN, Karsh J, Stahl NI, Blackwelder WC, Schiffman G, Klippel JH. Pneumococcal immunization in patients with systemic lupus erythematosus treated with immunosuppressives. J Rheumatol. 1985;12:1118021.

[11] Haut Conseil de la santé publique. Vaccination des personnes immunodéprimées ou aspléniques. Recommandations actualisées. Mais 2015. www.hcsp.fr/explore.cgi/avisrapportsdomaine?clefr $=504$

[12] Lindsey S, Oufnac B, Walker $H$. Safety of zoster vaccination administration in rheumatic patients on current biologic therapy. Arthritis Rheumatol. 2014;(66):S806.

51 [13] Naidus E, Damon L, Schwartz BS, Breed C, Liu C. Experience with use of Zostavax $\left({ }^{\circledR}\right)$ 52 in patients with hematologic malignancy and hematopoietic cell transplant recipients. Am 
J Hematol. 2012;87:123国5.

2 [14] Hibberd PL. Immunizations in solid organ transplant candidates and recipients -

3 UpToDate; 2017. www.uptodate.com/contents/immunizations-in-solid-organ-transplant4 candidates-and-recipients

[15] Adler S, Krivine A, Weix J, Rozenberg F, Launay O, Huesler J, et al. Protective effect of $\mathrm{A} / \mathrm{H} 1 \mathrm{~N} 1$ vaccination in immune-mediated disease--a prospectively controlled vaccination study. Rheumatology. 2012;51:695-700.

[16] Arad U, Tzadok S, Amir S, Mandelboim M, Mendelson E, Wigler I, et al. The cellular immune response to influenza vaccination is preserved in rheumatoid arthritis patients treated with rituximab. Vaccine. 2011;29:16430?8.

[17] Rehnberg M, Brisslert M, Amu S, Zendjanchi K, Håwi G, Bokarewa MI. Vaccination response to protein and carbohydrate antigens in patients with rheumatoid arthritis after rituximab treatment. Arthritis Res Ther. 2010;12:R111.

[18] van Assen S, Holvast A, Benne CA, Posthumus MD, van Leeuwen MA, Voskuyl AE, et al. Humoral responses after influenza vaccination are severely reduced in patients with rheumatoid arthritis treated with rituximab. Arthritis Rheum. 2010;62:75-81.

[19] Oren S, Mandelboim M, Braun-Moscovici Y, Paran D, Ablin J, Litinsky I, et al. Vaccination against influenza in patients with rheumatoid arthritis: the effect of rituximab on the humoral response. Ann Rheum Dis. 2008;67:937041.

[20] Bingham CO, Looney RJ, Deodhar A, Halsey N, Greenwald M, Codding C, et al. Immunization responses in rheumatoid arthritis patients treated with rituximab: results from a controlled clinical trial. Arthritis Rheum. 2010;62:64-74.

[21] Yusof MY, Vital EM, Buch MH. B cell therapies, approved and emerging: a review of infectious risk and prevention during use. Curr Rheumatol Rep. 2015;17:65.

[22] Nordøy T, Aaberge IS, Husebekk A, Samdal HH, Steinert S, Melby H, et al. Cancer patients undergoing chemotherapy show adequate serological response to vaccinations against influenza virus and Streptococcus pneumoniae. Med Oncol. 2002;19:7198.

[23] Hoebe C, Vermeiren A, Dukers-Muijrers N. Revaccination with Fendrix® or HBVaxPro ${ }^{\circledR}$ results in better response rates than does revaccination with three doses of Engerix-B® in previous non-responders. Vaccine. 2012;30:673407.

[24] Haut Conseil de la santé publique. Vaccins contre les hépatites $A$ et $B$ : tensions d'approvisionnement. 2017. www.hcsp.fr/explore.cgi/avisrapportsdomaine?clefr=594

[25] Haut Conseil de la santé publique. Vaccination contre l'hépatite B : problématique des non-répondeurs.

Novembre

www.mesvaccins.net/textes/hcspr20141107_hepBnonrepondeurs_(1).pdf

[26] Contin C, Pitard V, Delmas Y, Pelletier $\bar{N}$, Defrance T, Moreau JF, et al. Potential role of soluble CD40 in the humoral immune response impairment of uraemic patients. Immunology. 2003;110:1-10.

[27] Beasley RP, Hwang LY, Lee GC, Lan CC, Roan CH, Huang FY, et al. Prevention of perinatally transmitted hepatitis $B$ virus infections with hepatitis $B$ immune globulin and hepatitis B vaccine. Lancet. 1983;2:1099?102.

[28] Haut Conseil de la santé publique. Guide pour l'immunisation en post-exposition : $\begin{array}{lll}\text { vaccination } & \text { et } & 2016 .\end{array}$ www.hcsp.fr/explore.cgi/avisrapportsdomaine?clefr $=548$

[29] Scharpé J, Evenepoel P, Maes B, Bammens B, Claes K, Osterhaus AD, et al. Influenza vaccination is efficacious and safe in renal transplant recipients. Am $J$ Transplant. 2008;8:332?7.

[30] Morelon E, Pouteil Noble C, Daoud S, Cahen R, Goujon-Henry C, Weber F, et al. Immunogenicity and safety of intradermal influenza vaccination in renal transplant patients who were non-responders to conventional influenza vaccination. Vaccine. 2010;28:6885?90.

[31] Manuel O, Humar A, Chen MH, Chernenko S, Singer LG, Cobos I, et al. 
1 Immunogenicity and safety of an intradermal boosting strategy for vaccination against 2 influenza in lung transplant recipients. Am J Transplant. 2007;7:2567072.

3 [32] Zamora I, Simon JM, Da Silva ME, Piqueras Al. Attenuated varicella virus vaccine in 4 children with renal transplants. Pediatr Nephrol. 1994;8:190]2. et al. Seroprevalence of antibodies against varicella-zoster virus and response to the varicella vaccine in pediatric renal transplant patients. Pediatr Transplant. 2005;9:19206.

[34] Weinberg A, Horslen SP, Kaufman SS, Jesser R, Devoll-Zabrocki A, Fleckten BL, et al. Safety and immunogenicity of varicella-zoster virus vaccine in pediatric liver and intestine transplant recipients. Am J Transplant. 2006;6:56508.

[35] Posfay-Barbe KM, Pittet LF, Sottas C, Grillet S, Wildhaber BE, Rodriguez M, et al. Varicella-zoster immunization in pediatric liver transplant recipients: safe and immunogenic. Am J Transplant. 2012;12:2974085.

[36] Kumar D, Unger ER, Panicker G, Medvedev P, Wilson L, Humar A. Immunogenicity of quadrivalent human papillomavirus vaccine in organ transplant recipients. Am J Transplant. 2013;13:241107.

[37] Günther M, Stark K, Neuhaus R, Reinke P, Schröder K, Bienzle U. Rapid decline of antibodies after hepatitis $A$ immunization in liver and renal transplant recipients. Transplantation. 2001;71:47709.

[38] Wyplosz B, Burdet C, François H, Durrbach A, Duclos-Vallée JC, Mamzer-Bruneel MF, et al. Persistence of yellow fever vaccine-induced antibodies after solid organ transplantation. Am J Transplant. 2013;13:2458[61.

Figure 1. Stratégie de vaccination contre l'hépatite $B$ en cas d'insuffisance rénale chronique. 


\section{Figure 1: Stratégie de vaccination contre l'hépatite B-n cas d'insuffisance rénale chronique}

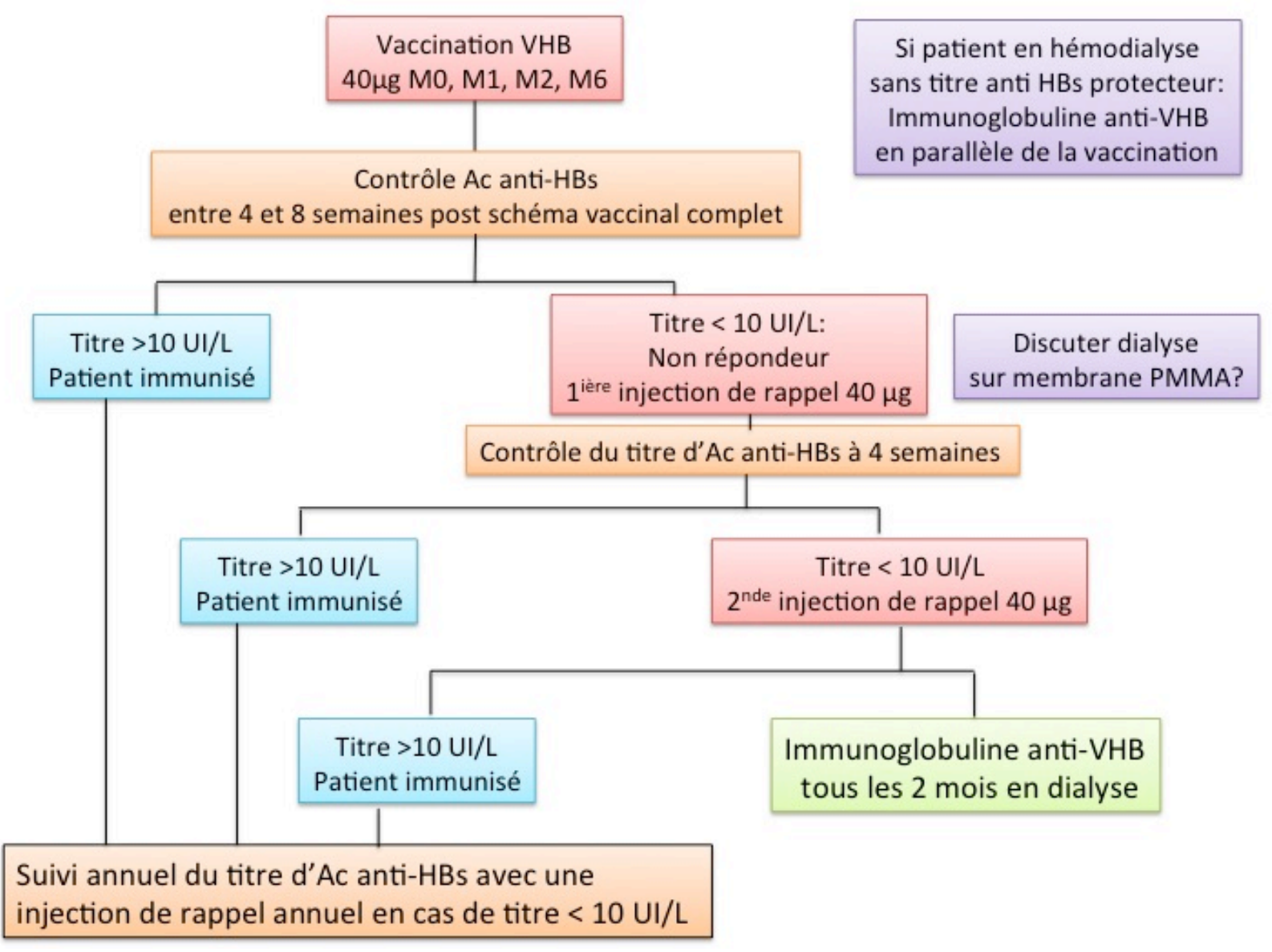


Tableau 1.

Effet de l'insuffisance rénale terminale sur les cellules de l'immunité (d'après [1]).

\begin{tabular}{|c|c|c|c|c|}
\hline \multicolumn{2}{|c|}{$\begin{array}{c}\text { Type } \\
\text { cellulaire } \\
\end{array}$} & \multirow[t]{2}{*}{ Rôle immunitaire } & \multicolumn{2}{|c|}{$\begin{array}{c}\text { Effet de l'urémie sur les différents types } \\
\text { cellulaires }\end{array}$} \\
\hline & & & Numération & Phénotype \\
\hline \multirow{3}{*}{ 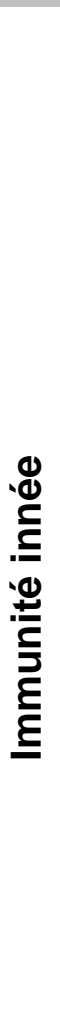 } & $\begin{array}{l}\text { Polynucléaires } \\
\text { neutrophiles }\end{array}$ & $\begin{array}{l}\text { Clairance } \\
\text { microbienne, } \\
\text { phagocytose } \\
\text { Nécrose tissulaire }\end{array}$ & $\lambda$ & $\begin{array}{l}\text { Surexpression des TLR2 et TLR4 } \\
\text { Sensibilité accrue à l'apoptose } \\
\text { Motilité et bactéricidie altérées } \\
\text { Augmentation des dérivés réactifs } \\
\text { de l'oxygène }\end{array}$ \\
\hline & Cellules NK & $\begin{array}{l}\text { Élimination } \\
\text { des cellules } \\
\text { tumorales ou viro- } \\
\text { infectées } \\
\text { Renforcement } \\
\text { des cellules } \\
\text { dendritiques }\end{array}$ & $y$ & $\begin{array}{l}\text { Surexpresssion du CD } 69 \\
\text { et du NKp44 } \\
\text { Sous-expression de NKG2D }\end{array}$ \\
\hline & $\begin{array}{l}\text { Monocytes et } \\
\text { macrophages }\end{array}$ & $\begin{array}{l}\text { Clairance } \\
\text { microbienne, } \\
\text { phagocytose } \\
\text { Présentation } \\
\text { antigénique }\end{array}$ & $\pi$ & $\begin{array}{l}\text { Surexpression des TLR } \\
\text { et intégrines } \\
\text { Réduction de la phagocytose } \\
\text { Diminution de la différenciation } \\
\text { en cellules dendritiques } \\
\text { Augmentation de la population } \\
\text { de monocytes pro-inflammatoires } \\
\text { CD14+CD16++ } \\
\text { Surexpression d'osteoactivin }\end{array}$ \\
\hline \multirow{4}{*}{ 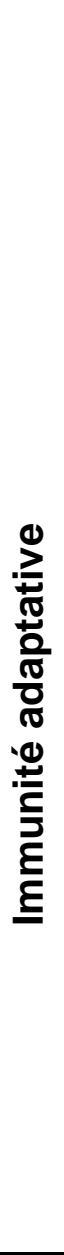 } & $\begin{array}{l}\text { Cellules } \\
\text { dendritiques }\end{array}$ & $\begin{array}{l}\text { Présentation } \\
\text { antigénique } \\
\text { Production } \\
\text { d'interféron } \alpha\end{array}$ & y & $\begin{array}{l}\text { Altération de la présentation } \\
\text { antigénique } \\
\text { Réduction de l'expression du CD86 }\end{array}$ \\
\hline & $\begin{array}{l}\text { Lymphocytes T } \\
\text { effecteurs }\end{array}$ & $\begin{array}{l}\text { Présentation } \\
\text { antigénique (CD4) } \\
\text { Élimination } \\
\text { des cellules } \\
\text { tumorales ou viro- } \\
\text { infectées (CD8) }\end{array}$ & עلע & $\begin{array}{l}\text { Surexpression des récepteurs } \\
\text { à l'IL-2 et au TNF } \\
\text { Réduction de la réponse } \\
\text { aux stimulations cytokiniques } \\
\text { Différentiation cellulaire accrue } \\
\text { Sensibilité accrue à l'apoptose } \\
\text { Raccourcissement des télomères } \\
\text { Réduction des populations } \\
\text { de lymphocytes T naïfs } \\
\text { Augmentation des populations } \\
\text { de lymphocytes T CD4+ CD28- } \\
\text { pro-inflammatoires } \\
\text { Altération de la réponse } \\
\text { antigénique spécifique }\end{array}$ \\
\hline & $\begin{array}{l}\text { Lymphocytes } T \\
\text { régulateurs }\end{array}$ & $\begin{array}{l}\text { Suppression } \\
\text { de la réponse } \\
\text { effectrice } \\
\text { lymphocytaire T }\end{array}$ & y & Altération de la fonction régulatrice \\
\hline & Lymphocytes B & $\begin{array}{l}\text { Présentation } \\
\text { antigénique } \\
\text { Réponse } \\
\text { humorale, }\end{array}$ & y & $\begin{array}{l}\text { Réduction des populations } \\
\text { de lymphocytes B naïfs et mémoire } \\
\text { Diminution de l'expression de BAFF } \\
\text { et Bcl-2 }\end{array}$ \\
\hline
\end{tabular}


TLR : toll like receptor; IL-2 : interleukine 2 ; TNF : tumor necrosis factor; BAFF : B-cell activating factor, ou "tumor necrosis factor ligand superfamily member 13B"; Bcl2 : BCL2 apoptosis regulator 
Tableau 2.

Résumé des vaccinations conseillées en cas de maladie rénale hors transplantation, en addition aux recommandations pour la population générale.

\begin{tabular}{|c|c|c|}
\hline & Vaccins recommandés & Vaccins contre-indiqués \\
\hline $\begin{array}{l}\text { Syndrome } \\
\text { néphrotique }\end{array}$ & $\begin{array}{l}\text { Pneumocoque : Prevenar13 } 3^{\circledR} \text { suivi } \\
\text { par Pneumo23 }{ }^{\circledR} \text { à } 8 \text { semaines. } \\
\text { Rappel par Pneumo23 }{ }^{\circledR} \text { à } 5 \text { ans } \\
\text { Grippe saisonnière, vaccination } \\
\text { annuelle } \\
\text { DTP : identique à la population } \\
\text { générale }\end{array}$ & $\begin{array}{l}\text { Aucun (sauf en cas } \\
\text { de traitement } \\
\text { immunosuppresseur, } \\
\text { voir infra) }\end{array}$ \\
\hline $\begin{array}{l}\text { Traitement } \\
\text { immunosuppresseur }\end{array}$ & 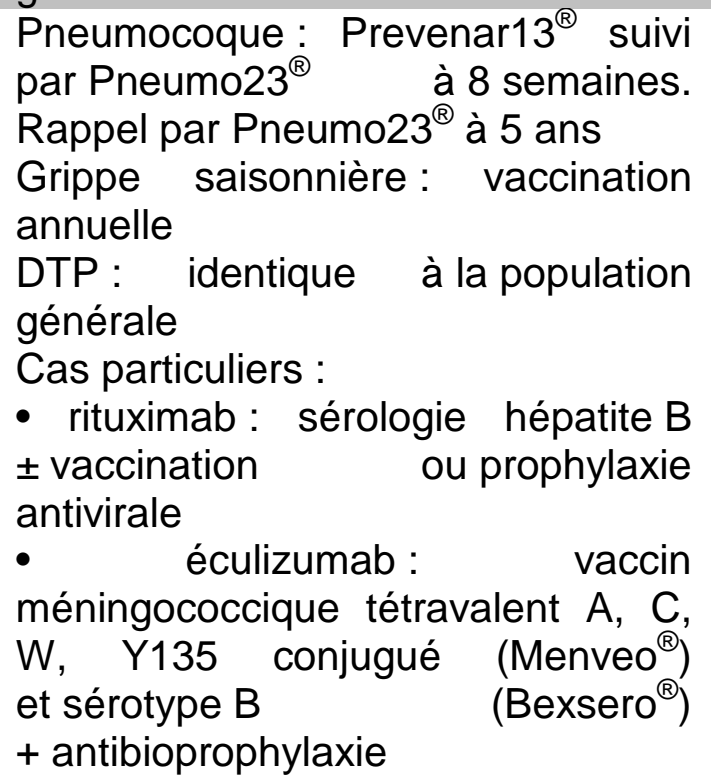 & $\begin{array}{l}\text { BCG } \\
\text { Fièvre jaune } \\
\text { ROR } \\
\text { Varicelle } \\
\text { Zona }\end{array}$ \\
\hline
\end{tabular}

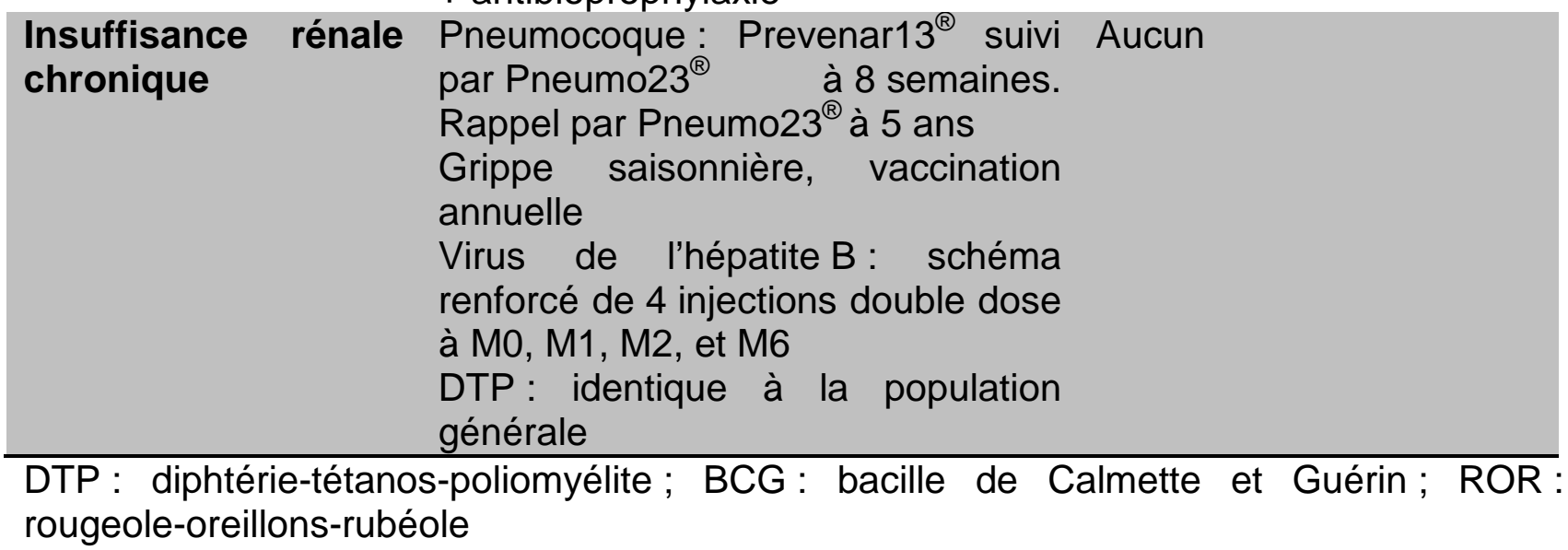


Tableau 3.

Résumé des vaccinations recommandées chez les patients en attente de transplantation et après transplantation [11].

\begin{tabular}{|c|c|c|}
\hline & Vaccins recommandés & $\begin{array}{l}\text { Vaccins } \\
\text { contre- } \\
\text { indiqués }\end{array}$ \\
\hline $\begin{array}{l}\text { Évaluation } \\
\text { Pré- } \\
\text { transplantation }\end{array}$ & 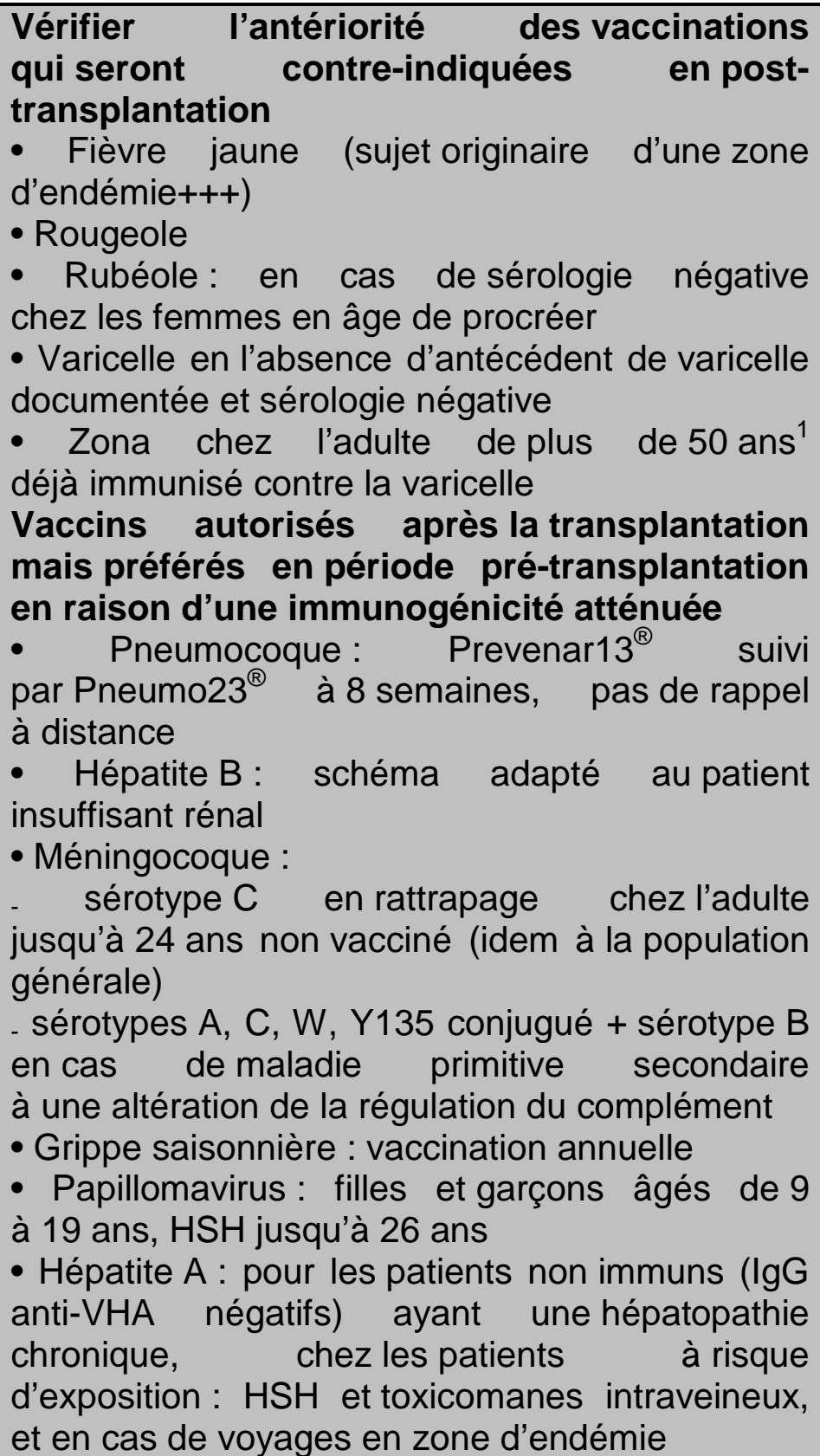 & $\mathrm{BCG}$ \\
\hline $\begin{array}{l}\text { Post- } \\
\text { transplantation }\end{array}$ & $\begin{array}{l}\text { Pneumocoque : } \quad \text { Prevenar } 13^{\circledR} \\
\text { par Pneumo23 } \\
\text { Grippe saisonnière : vaccination annuelle } \\
\text { DTP }\end{array}$ & $\begin{array}{l}\text { BCG } \\
\text { Fièvre jaune } \\
\text { ROR } \\
\text { Varicelle } \\
\text { Zona }\end{array}$ \\
\hline
\end{tabular}

BCG : bacille de Calmette et Guérin ; DTP : diphtérie-tétanos-poliomyélite ; HSH : homme ayant des relations sexuelles avec des hommes

${ }^{1}$ L'âge à partir duquel il est raisonnable de proposer une vaccination contre le zona reste sujet à discussion. Ce vaccin est recommandé à partir de 65 ans dans la population générale, mais certains auteurs proposent de vacciner dès l'âge de 50 ans avant l'instauration d'un traitement immunosuppresseur 